\title{
Excitation and damping of slow magnetosonic standing waves in a solar coronal loop
}

\author{
M. Selwa ${ }^{1}$, K. Murawski ${ }^{1}$, and S. K. Solanki ${ }^{2}$ \\ ${ }^{1}$ Institute of Physics, UMCS, ul. Radziszewskiego 10, 20-031 Lublin, Poland \\ e-mail: mselwa@kft.umcs.lublin.pl \\ 2 Max-Planck-Institut für Sonnensystemforschung, Max-Planck-Str. 2, 37191 Katlenburg-Lindau, Germany
}

Received 5 November 2004 / Accepted 24 January 2005

\begin{abstract}
We consider slow magnetosonic standing waves that are impulsively excited in a solar coronal loop. The onedimensional numerical model we implement includes the effects of nonlinearity, optionally thermal conduction, heating, and cooling of the solar plasma. We numerically evaluate excitation and damping times of a standing wave in hot coronal loops on the basis of a parametric study. Results of the numerical simulations reveal that initially launched impulses mainly trigger the fundamental mode and its first harmonic, depending on the location of these pulses in space. Parametric study shows that these standing waves are excited in a dozen or so wave periods corresponding roughly to $13 \mathrm{~min}$ and that they are strongly damped over a similar time-scale.
\end{abstract}

Key words. Sun: corona - Sun: magnetohydrodynamics (MHD) - Sun: oscillations

\section{Introduction}

Coronal loop oscillations have recently become a subject of considerable observational and theoretical interest. Impulsively generated, standing slow waves in hot $(T>6 \mathrm{MK})$ loops have been detected with SOHO/SUMER (Wang et al. 2003a). It is interesting that these standing waves are strongly attenuated while the loops cool down (Curdt et al. 2003). Observations from the SUMER and EIT on SOHO and TRACE have revealed that Yohkoh loop oscillations often seem to be triggered by micro- or sub-flares near a loop foot-point (Wang et al. 2003b). In such cases the trigger may be plasma flow injected into the loop from one foot-point.

Several attenuation mechanisms have been proposed: wave leakage into the chromosphere (Ofman 2002; Van Doorsselaere et al. 2004), lateral wave leakage due to curvature of loops (Roberts 2000), phase mixing (Nakariakov 1999; Ofman \& Aschwanden 2002), resonant absorption (Ruderman \& Roberts 2002), and non-ideal MHD effects (Roberts 2000). In particular, Ofman \& Wang (2002) have found that thermal conduction leads to rapid damping of slow standing waves, with a less significant contribution from compressive viscosity (Ofman et al. 2002a). Ofman et al. (2002b) have shown that a nonlinear steepening of slow waves leads to their enhanced dissipation. Nakariakov et al. (2000a) have found that dissipation and stratification are the main factors influencing slow wave evolution, while De Moortel et al. (2002a) have deduced that thermal conduction can account for the observed damping times.

In another study, Nakariakov et al. (2004) and Tsiklauri et al. (2004) demonstrated that in a coronal loop an impulsive energy release efficiently excites the second spatial harmonic. The considered model included the effects of gravitational stratification, heat conduction, radiative losses, external heat input, and Braginskii bulk viscosity. An extensive review of longitudinal intensity fluctuations observed in coronal loops is presented by De Moortel et al. (2002b).

Our work is aimed at an explanation of the excitation mechanism of slow standing waves, while including main damping mechanism, i.e. thermal conduction. We discuss impulsively generated waves that are described by fully nonlinear one-dimensional ideal MHD equations. As a consequence of this one-dimensional assumption, the Alfvén and fast magnetosonic waves are removed from the physical system, which only contains the slow waves.

\section{A numerical model}

As we only pay attention to slow waves, we consider that the coronal loop lies along the $x$-direction. Additionally we assume that velocity $\boldsymbol{V}=[V, 0,0]$, magnetic field $\boldsymbol{B}=[B, 0,0]$, and the plasma quantities only depend on time $t$ and coordinate $x$. As a consequence of these assumptions we adopt the one-dimensional MHD equations:

$$
\begin{array}{r}
\frac{\partial \varrho}{\partial t}+\frac{\partial(\varrho V)}{\partial x}=0, \\
\varrho\left(\frac{\partial V}{\partial t}+V \frac{\partial V}{\partial x}\right)=-\frac{\partial p_{\mathrm{T}}}{\partial x}, \\
\frac{\partial(\varrho E)}{\partial t}+\frac{\partial\left[\left(\varrho E+p_{\mathrm{T}}\right) V\right]}{\partial x}=\frac{\partial}{\partial x}\left(\sigma \frac{\partial T}{\partial x}\right)+\mathcal{H}-\mathcal{L}, \\
\frac{\partial B}{\partial t}=\frac{\partial B}{\partial x}=0 .
\end{array}
$$


Here $\varrho$ is the mass density, $T$ - the temperature, and the symbol $p_{\mathrm{T}}$ denotes a total pressure that represents the sum of the gas and magnetic pressures:

$p_{\mathrm{T}}=\left(p+\frac{B^{2}}{2 \mu}\right)$

where $\mu$ is the magnetic permeability. $E$ is the plasma energy density:

$E=\frac{\varrho V^{2}}{2}+\frac{p_{\mathrm{T}}}{\gamma-1}$

where $\gamma$ is the adiabatic index. Coefficient $\sigma$ corresponds to the thermal conductivity that follows from the formulation of Spitzer (1962)

$\sigma=\kappa T^{5 / 2}$,

where $\kappa=9.2 \times 10^{-7} \mathrm{erg} \mathrm{cm}^{-1} \mathrm{~s}^{-1} \mathrm{~K}^{-7 / 2}$ is the plasma thermal conductivity.

In Eq. (3) $\mathcal{L}$ represents a cooling term due to radiative losses from an optically thin plasma (Raymond 1978) and $\mathcal{H}$ is the heating term that is implemented phenomenologically as (Zingale et al. 2002)

$\mathcal{H}=\mathcal{H}_{0} \exp \left[-\frac{\left(x-x_{0}\right)^{2}}{2 w_{\mathrm{h}}^{2}}\right] \exp \left(-\frac{t}{\tau}\right)$.

We choose and fix the width of the heating source $w_{\mathrm{h}}=1.25 \times$ $10^{8} \mathrm{~cm}, \mathcal{H}_{0}=0.2 \operatorname{erg~cm}^{-3} \mathrm{~s}^{-1}$, the duration $\tau=41 \mathrm{~s}$, while the spatial position of heat deposition $x_{0}$ is left as a free parameter. These values correspond to parameters of a typical solar flare according to Nakariakov et al. (2004).

\subsection{The loop equilibrium}

We consider the equilibrium at which pressure $p_{0}=c_{\mathrm{s}}^{2} \varrho_{0} / \gamma=$ const. and plasma is at rest, $V_{0}=0$. We choose equilibrium density profile $\varrho_{0}$, which varies with $x$ such that $\varrho_{0}$ attains large values at loop foot-points settled at $x=0$ and $x=L$, where $L=50 \times 10^{8} \mathrm{~cm}$ is the loop length which corresponds to loop radius of $13-16 \mathrm{Mm}$. The loop is relatively short because short loops are easier to simulate. In Sect. 3.2 we also consider loops with other lengths. The mass density profile $\varrho_{0}(x)$ is similar to the profile implemented by Ofman (2002), i.e.

$\varrho_{0}(x)=\varrho_{\mathrm{c}}\left\{\frac{d}{2}\left[\tanh \left(s\left(x-x_{\mathrm{tr}}\right) \cdot\left(x-L+x_{\mathrm{tr}}\right)\right)+1\right]+1\right\}$.

Here $d$ is the ratio of the photospheric mass density $\varrho_{\mathrm{ph}}$ to coronal mass density $\varrho_{\mathrm{c}}=10^{-15} \mathrm{~g} \mathrm{~cm}^{-3}$. We allow this parameter to vary in the range $10^{4} \leq d \leq 10^{8}$. The quantity $s=0.5 \times 10^{-17} \mathrm{~cm}^{-1}$ denotes the slope of $\varrho_{0}(x)$ at the loop footpoints, and $x_{\mathrm{tr}}=0.2842 \times 10^{8} \mathrm{~cm}$ corresponds to the position of the transition region. According to Eq. (9) $\varrho_{0}$ jumps at this location. As $T_{0}(x) \sim p_{0} / \varrho_{0}(x)$, the plasma temperature $T_{0}$ is higher at the solar corona than at the foot-points. We also choose the sound speed in the solar corona $c_{\mathrm{s}}=\sqrt{\gamma p_{0} / \varrho_{0}(x=L / 2)}=$ $0.35 \times 10^{8} \mathrm{~cm} \mathrm{~s}^{-1}$.

\subsection{Perturbations}

For the coronal loop that is described by Eqs. (1)-(3), perturbations can be excited in such a loop in numerous ways. Here we focus our attention on impulsively excited waves. We launch a hot pulse in the mass density, pressure, and velocity. This pulse has the following form:

$$
\begin{aligned}
& \delta \varrho(x, t=0)=A_{\varrho} \exp \left[-\left(x-x_{0}\right)^{2} / w^{2}\right], \\
& \delta p(x, t=0)=A_{\mathrm{p}} \exp \left[-\left(x-x_{0}\right)^{2} / w^{2}\right], \\
& \delta V(x, t=0)=A_{\mathrm{V}} \exp \left[-\left(x-x_{0}\right)^{2} / w^{2}\right],
\end{aligned}
$$

where $A_{\varrho}, A_{\mathrm{p}}$, and $A_{\mathrm{V}}$ are initial amplitudes of the pulse, $x_{0}$ its initial position, and $w$ its initial width.

\subsection{Analytical formulae for standing waves}

To derive analytical formulae for standing waves, we replace the profile of Eq. (9) by a uniform profile $\varrho_{0}=$ const., then neglect thermal conduction, heating, and cooling by setting $\sigma=\mathcal{H}=\mathcal{L}=0$ in Eq. (3). Additionally we assume solidwall boundary conditions at $x=0$ and $x=L$ by setting $V=0$ there. In this case initial perturbations after a sufficiently long time excite the standing wave oscillations given by the analytical formulae:

$$
\begin{aligned}
V(x, t) & =A_{\mathrm{v}} \cos \left(\omega_{n} t\right) \sin \left(k_{n} x\right), \\
\delta \varrho(x, t) & =-\frac{\varrho_{0} A_{\mathrm{v}}}{c_{\mathrm{s}}} \sin \left(\omega_{n} t\right) \cos \left(k_{n} x\right), \\
\delta p(x, t) & =-\varrho_{0} A_{\mathrm{v}} c_{\mathrm{s}} \sin \left(\omega_{n} t\right) \cos \left(k_{n} x\right),
\end{aligned}
$$

where:

$k_{n}=\frac{n \pi}{L}, \quad \omega_{n}=c_{\mathrm{s}} k_{n}$.

Notice that perturbed mass density $\delta \varrho$ and velocity $V$ display a phase shift of a quarter wave period.

\section{Numerical results}

In this part of the paper we present numerically obtained results for various run parameters. The plasma equations are solved numerically with the FLASH code (Fryxell et al. 2000). For most numerical runs 300 blocks are chosen. Each block contains 8 grid cells. We performed grid convergence studies to verify that the numerical diffusion is negligibly small. Free boundary conditions are used at the boundaries of the simulation region, and all physical quantities are measured in cgs units.

We discuss the fundamental mode and the first harmonic, which are both triggered by temperature perturbations and go on to pay particular attention to excitation and damping times of these modes. Our simulations show that these lowest modes are the dominantly excited ones for the pulse parameters employed.

\subsection{Excitation by temperature perturbation}

We now consider the case of ideal plasma for which there is no thermal conduction, cooling, and heating. This implies that 

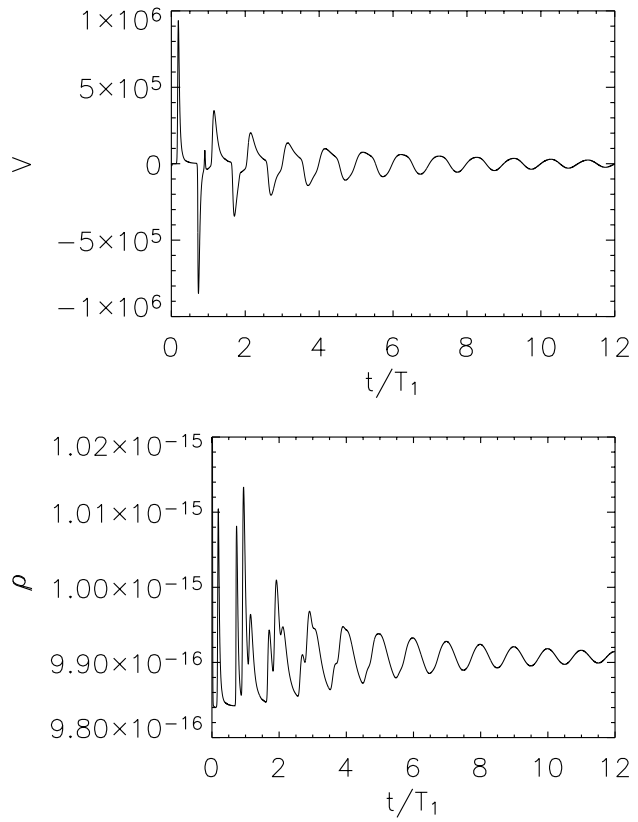

Fig. 1. Evolution of velocity $V(x=L / 4, t)$ (top panel) and mass density $\varrho(x=L / 4, t)$ (bottom panel) for mass density contrast $d=10^{8}$, pulse width $w=L / 40$, and initial pulse position $x_{0}=L / 4$.

the source terms on the right hand side of Eq. (3) are set to zero. So, we have $\sigma=\mathcal{L}=\mathcal{H}=0$. Additionally we assume that there are no initial velocity perturbations. In this case slow waves are excited solely by temperature perturbations given by Eqs. (10)-(12) with $A_{\varrho}=0.125 \varrho_{0}\left(x_{0}\right), A_{\mathrm{p}}=0.25 p_{0}$, and $A_{\mathrm{V}}=$ 0 . We launch the pulses of Eqs. (10)-(11) with a width of $w=$ $L / 40$, which is a typical value determined from observations (Nakariakov et al. 2004). We also carry out a parametric study in which parameters are allowed to vary around the adopted fiducial values. First we consider in some detail three cases that correspond to different positions of the initial pulses: (a) $x_{0}=$ $L / 4$; (b) $x_{0}=L / 2$; (c) $x_{0}=0$.

\subsubsection{Time evolution of the pulses}

The basic time-signature of the excited mode is similar to a damped oscillation (see Fig. 1). However, the wave form evolves significantly with time. At the initial stages of evolution and in addition to the signal that is typical of standing mode, we observe some short-wave oscillations (Fig. 1), also seen in FFT spectra - not shown - and in wavelet spectra (Fig. 4). At later times the signal becomes almost sinusoidal.

\subsubsection{Pulses at a quarter of the loop length and at the apex of the loop}

If the initial pulse is launched at $x_{0}=L / 4\left(x_{0}=L / 2\right)$, the fundamental (first harmonic) mode is excited. Figure 2 displays spatial profiles of these waves at given moments in time. As the fundamental standing wave is twice longer than its harmonic (Fig. 2), the fundamental wave is less sensitive to the inhomogeneous medium, and essentially it occupies the cavity over all its length $L$. The harmonic wave is more sensitive to the
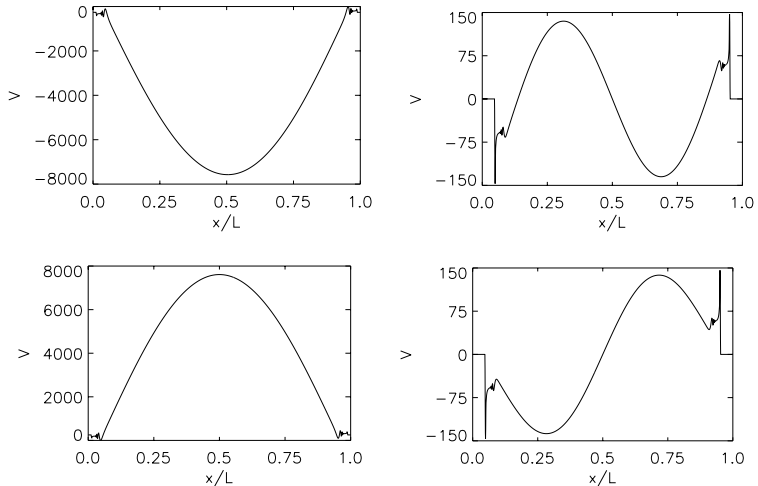

Fig. 2. Spatial profiles of velocity $V\left(x, t=18.842 T_{1}\right)$ (left top panel), $V\left(x, t=19.274 T_{1}\right)$ (left bottom panel), $V\left(x, t=37.841 T_{2}\right)$ (right top panel), and $V\left(x, t=38.281 T_{2}\right)$ (right bottom panel) for mass density contrast $d=10^{8}$, pulse width $w=L / 40$, pulse position $x_{0}=L / 4$ (left panels), and $x_{0}=L / 2$ (right panels). Left (right) profiles correspond to the fundamental (first harmonic) mode.
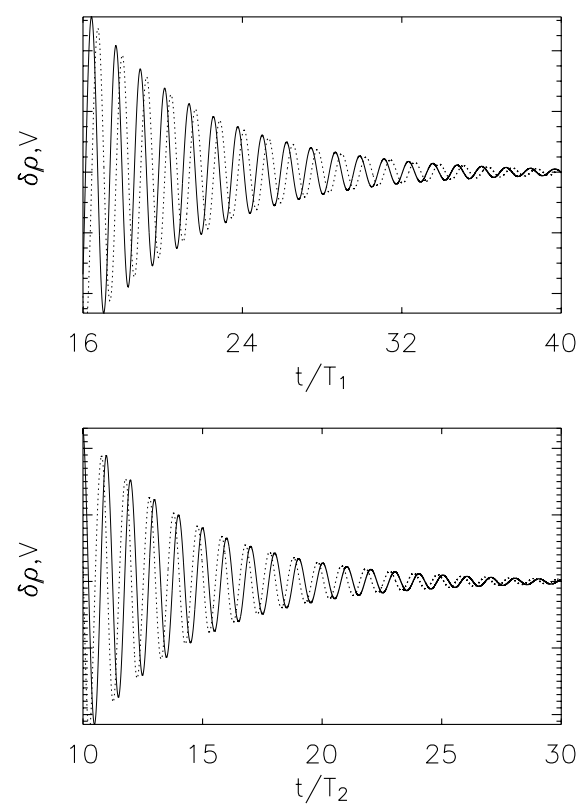

Fig. 3. Time-signatures of perturbed mass density $\delta \varrho(x=L / 4, t)$ (solid line) and velocity $V(x=L / 4, t)$ (dashed line) for $d=10^{8}, w=L / 40$, $x_{0}=L / 4$ (top panel), and $x_{0}=L / 2$ (bottom panel).

inhomogeneity than the fundamental wave, resulting in enhanced energy leakage into the photosphere.

Time-signatures of perturbed mass density $\delta \varrho$ and velocity $V$, which are detected at spatial point $x=L / 4$, are shown in Fig. 3 for a time after the initial high frequency power has died away. Oscillation amplitudes decrease with time as a consequence of wave damping due to energy leakage into the photosphere.

In all considered cases the presence of the standing wave in the system is evaluated on the basis of normalized phase shift $\delta \phi$, for the fundamental $(n=1)$ or harmonic $(n=2)$ standing waves. We establish an excitation criterion according to which a standing wave is present in the system if $\delta \phi$ departs 

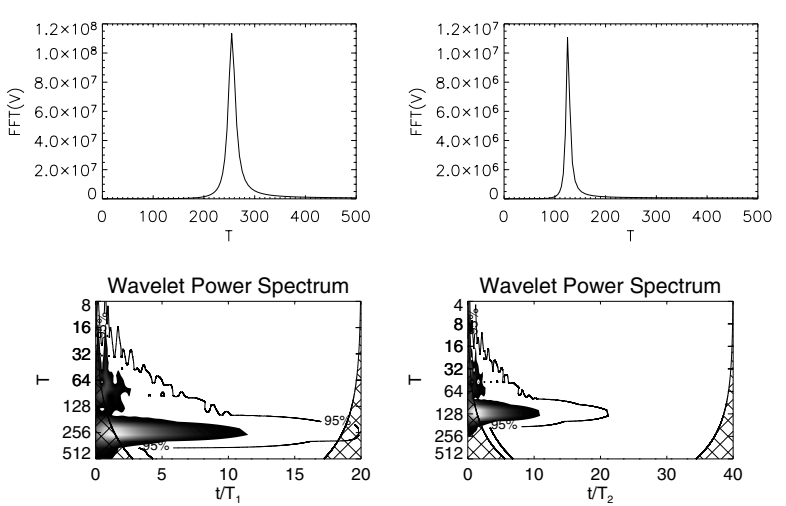

Fig. 4. Spectra of velocity $V(x=L / 4, t)$ from Fig. 3 . The Fourier spectra are displayed in the top panels and the corresponding wavelet spectra in the bottom panels. Both the Fourier and wavelet analysis lead to wave periods $T_{1} \approx 254 \mathrm{~s}, T_{2} \approx 125 \mathrm{~s}$, where $T_{n}$ is the $n$th standing wave period. The hatched area in the lower frames indicate regions where the wavelet transform is less accurate.

by $20 \%$ from $1 / 4$, since the quarter period lag is a signature of the analytical solution of Sect. 2.3 , viz.

$$
\frac{1}{4} \cdot 80 \% \leq \delta \phi \leq \frac{1}{4} \cdot 120 \% \text {. }
$$

This criterion is fulfilled for $t>t_{\min }$. As the standing wave excitation time we assume $t_{\min }$. According to this criterion the fundamental (harmonic) standing wave is excited at $t \approx 6 T_{1}(t \approx$ $3.5 T_{2}$ ), where $T_{1}$ and $T_{2}$ are the analytically evaluated periods that are expressed by

$T_{n}=\frac{2 L_{\mathrm{eff}}}{n c_{\mathrm{s}}}$

where $n$ denotes the standing wave number, $c_{\mathrm{s}}-$ the sound speed, and $L_{\text {eff }}-$ the effective loop length. From Fig. 2 we estimate that $L_{\mathrm{eff}} \approx 45 \times 10^{8} \mathrm{~cm}$ and then $T_{1} \approx 257 \mathrm{~s}$ and $T_{2} \approx 128 \mathrm{~s}$. These values are close to the results of the fast Fourier transform (FFT) and wavelet analyses (Fig. 4). Small differences result from the fact that the effective length of the cavity is lower than $L$ and differs for the case of $n=1$ and $n=2$. Moreover, $c_{\mathrm{s}}$ is an inhomogeneous function of $x$ in our simulations, but is assumed to be $x$-independent when evaluating Eq. (18).

We have restricted the Fourier analysis to $t>3 T_{1}$ in order to remove the transient signal at the initial stage of temporal evolution. This signal is discernible in the wavelet power spectrum (Fig. 4) obtained using a Morlet mother function (Torrence \& Compo 1998)

$\psi(\eta)=\pi^{-1 / 4} \mathrm{e}^{\mathrm{i} m \eta} \mathrm{e}^{-\eta^{2} / 2}$

with $m=6$. The wavelet transform was applied to the full time series. The solid contour represents $95 \%$ confidence, and the shaded area $90 \%$.

\subsubsection{A pulse at a foot-point}

The initial pulse of Eqs. (10)-(11) is launched at the loop footpoint located at $x=0$. Figure 5 displays the corresponding
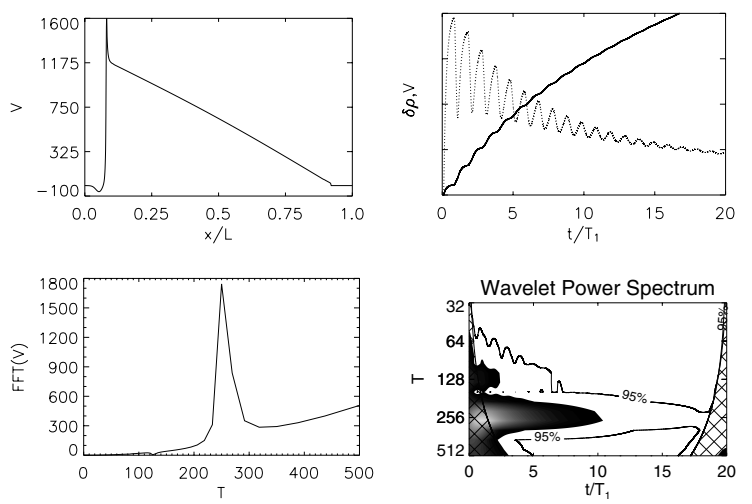

Fig. 5. A spatial velocity profile at $t \approx 20 T_{1}$ (left top panel), timesignatures (right top panel) of the perturbed mass density $\delta \varrho(x=$ $L / 4, t)$ (solid line), and velocity $V(x=L / 4, t)$ (dotted line) for $d=10^{8}$, $w=L / 40$, and $x_{0}=0$. The corresponding Fourier and wavelet power spectra are displayed in the bottom panels.

results. As a result of a hot initial pulse, plasma is heated locally at the loop foot-point. Just warmed-up and dense photospheric plasma leaves the excitation region and fills up the coronal loop. As a consequence, $\delta \varrho$ grows in time (top right panels). The mean flow is directed to the loop center, and its efficiency declines both spatially in the region $x>x_{0}$ (left top panel) and with time (right top panel). Velocity power spectra (bottom panels) reveal the wave period of the fundamental standing wave $T_{1}$. The background in the Fourier power spectrum is introduced by the velocity drift. The wave excitation time $t_{\mathrm{ex}} \approx 3 T_{1}$, and the damping time $\tau \approx 5 T_{1}$. Qualitatively similar results have been obtained in the case of the initial pulse launched in the photosphere at $x_{0}=-L / 50$, i.e. outside the loop, corresponding to subsurface layers of the Sun (not shown). Recently Tsiklauri et al. (2004) discussed the case of heat deposition at a loop-foot point.

\subsubsection{Excitation of a packet of standing waves}

We excite different waves by setting the initial pulses in different parts of the loop. Fourier spectra show that an almost pure first harmonic standing wave is excited when the initial pulse is launched at the loop apex. In some cases we do not observe a single standing mode but instead a packet of modes in which the fundamental and harmonic standing modes make the largest contribution. Moving the excitation point from the loop apex to the foot-points results in an excitation of the fundamental mode. For the excitation points that are close to $x_{0}=L / 2$ we observe that the Fourier power in the first harmonic standing mode is much higher than in the fundamental standing mode, while for $x_{0}=3 L / 8$ the scenario changes and the fundamental mode dominates (Fig. 6).

\subsection{Parametric studies of wave excitation and damping times}

In this part of the paper we present results of parametric studies. We vary several parameters, such as pulse position $x_{0}$, density contrast $d$, pulse width $w$, temperature of initial pulses, and 

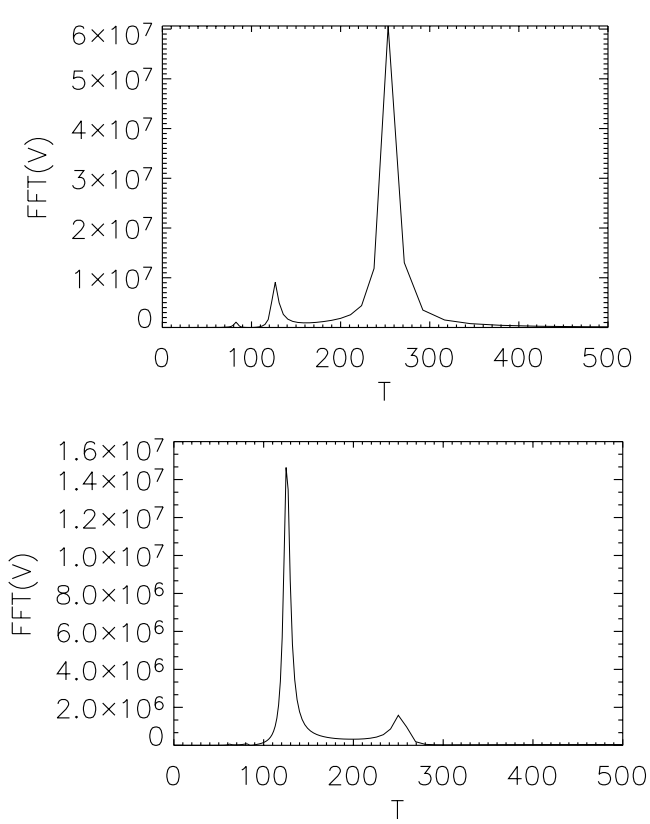

Fig. 6. Fourier spectra of the velocity $V(x=L / 4, t)$ for $d=10^{8}$, $w=L / 40, x_{0}=0.375 L$ (top panel), and $x_{0}=0.475 L$ (bottom panel). Fundamental and the first harmonic standing modes are dominant in the top and bottom panels, respectively, but the first harmonic and fundamental modes are present there too.

perturbations in velocity $A_{\mathrm{V}}$. We analyze how the standing wave excitation time $t_{\mathrm{ex}}$ and damping time $\tau$ depend on these parameters.

We first filter out non-oscillatory components from the time-signatures $\varrho\left(x_{0}=L / 4, t\right)$ and $V\left(x_{0}=L / 4, t\right)$ to remove trends, such as seen in the upper right panel of Fig. 5, and to get a pure signal that corresponds solely to oscillations. The filtering is done by the code originally developed by Ofman (2002). We estimate excitation time $t_{\mathrm{ex}}$ with the criterion described by Eq. (17), while for damping time $\tau$ we fit an envelope of a velocity profile into the following formula

$V\left(x=x_{\mathrm{d}}, t\right)=V_{0} \exp \left(-\frac{t-t_{\mathrm{ex}}}{\tau}\right), t \geq t_{\mathrm{min}}$,

where $x_{\mathrm{d}}$ denotes the detection point, and $V_{0}$ is the amplitude of the velocity $V$ at time $t_{\text {ex }}$.

We first consider the dependence of $t_{\mathrm{ex}}$ and $\tau$ on the location $x_{0}$ of the initial pulse. Figure 7 shows that the fundamental (harmonic) standing wave excitation time $t_{\mathrm{ex}}$ generally grows (declines) as the pulse position $x_{0}$ moves from the loop footpoint $\left(x_{0}=0\right)$ to its apex $\left(x_{0}=L / 2\right)$. In the case of the fundamental standing wave, the damping time depends weakly on $x_{0}$ with a slight tendency to decrease with $x_{0}$ (bottom panel of Fig. 7). The second standing wave damping time decreases as $x_{0}$ approaches $L / 2$.

These results support evidence that excitation and damping times are not constant but depend on the location of the initial pulse. Excitation time varies from its lowest value of $3 T_{1}$ for $x_{0}=0$ to its highest value of $16 T_{1}$ for $x_{0}=0.45 L$ for the fundamental standing wave (and from $3 T_{2}$ for $x_{0}=L / 2$ to $8 T_{2}$ for $x_{0}=0.45 \mathrm{~L}$ in the case of the second standing wave). The obtained values of damping time are in the
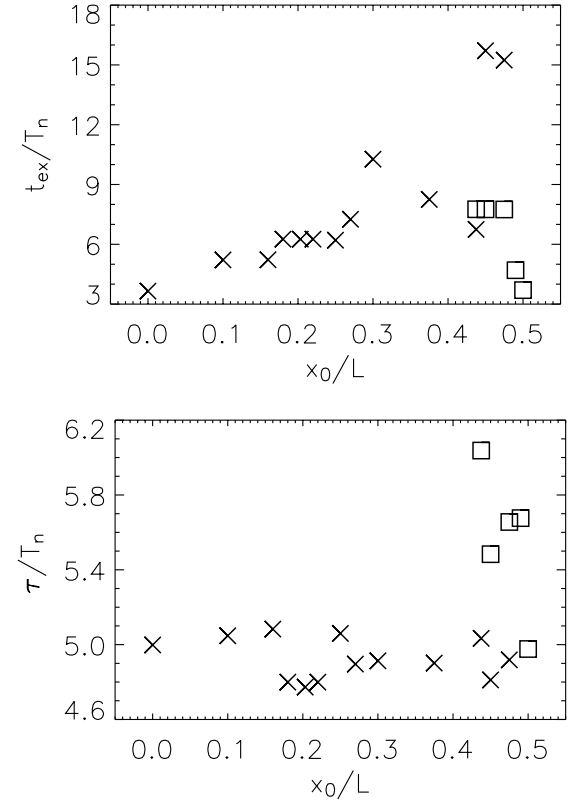

Fig. 7. Normalized standing wave excitation time $t_{\mathrm{ex}} / T_{n}$ (top panel) and normalized damping time $\tau / T_{n}$ (bottom panel) vs. normalized pulse position $x_{0} / L$ for $d=10^{8}$ and $w=L / 40$. The squares (crosses) correspond to the fundamental (harmonic) standing waves.

range $4.7 T_{1}<\tau<5.2 T_{1}$ for the fundamental standing wave and $5 T_{2}<\tau<6.1 T_{2}$ for the second standing wave, for which the lowest (highest) value is obtained for $x_{0}=L / 2$ (for $x_{0}=0.44 L$ ). Note that $T_{1} \approx 2 T_{2}$, so that in absolute terms the 2nd wave is damped more rapidly than the fundamental wave.

We conclude from the above results that the fundamental (harmonic) standing wave is excited most efficiently by an initial pulse located in the neighborhood of a loop foot-point (of the loop apex). At these points the excitation (damping) time of these waves is equal to 3 (5) periods, corresponding roughly to $13 \mathrm{~min}$ (11 $\mathrm{min})$.

The standing wave excitation time varies with density contrast $d$ (Fig. 8). For high values of $d$ the fundamental standing wave is excited less efficiently than for low values, for which $t_{\mathrm{ex}}$ attains its minimum; the excitation time is doubled. At the smallest (largest) considered value of $d=10^{2}\left(d=10^{8}\right)$ the damping time is $2.5 T_{1}\left(6.2 T_{1}\right)$. From these results we conclude that the fundamental standing wave is excited faster in low density contrast regions, as such a structure is more susceptible to energy leakage at the foot-points. As a consequence, it is easier for a perturbation to adjust to standing waves profiles. Obviously, damping of the wave is enhanced and the damping time is of the order of a few wave periods. Damping is about twice weaker at $d=10^{8}$ than at $d=10^{2}$, although the dependence of $\tau$ on $d$ is complex.

The standing wave excitation time depends on pulse width $w$ (Fig. 9). For a sufficiently wide pulse the fundamental standing wave is excited faster by wider pulses, although there is significant scatter (top panel). The damping time oscillates around a value of about $4.8 T_{1}$, within a relatively narrow range implying that it is almost independent of $w$. These results are a consequence of the fact that wider pulses are closer to the sine 

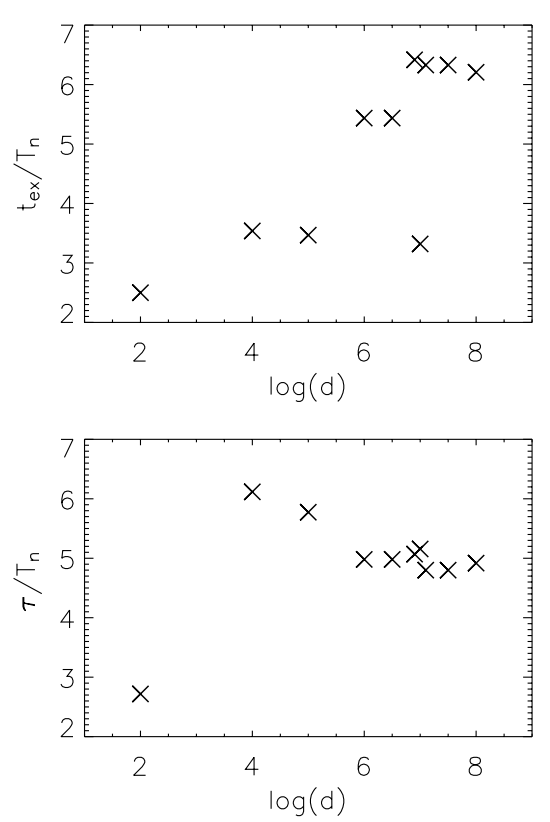

Fig. 8. Normalized fundamental standing wave excitation time (top panel) and normalized damping time (bottom panel) vs. $\log d$ for $w=$ $L / 40$ and $x_{0}=L / 4$.
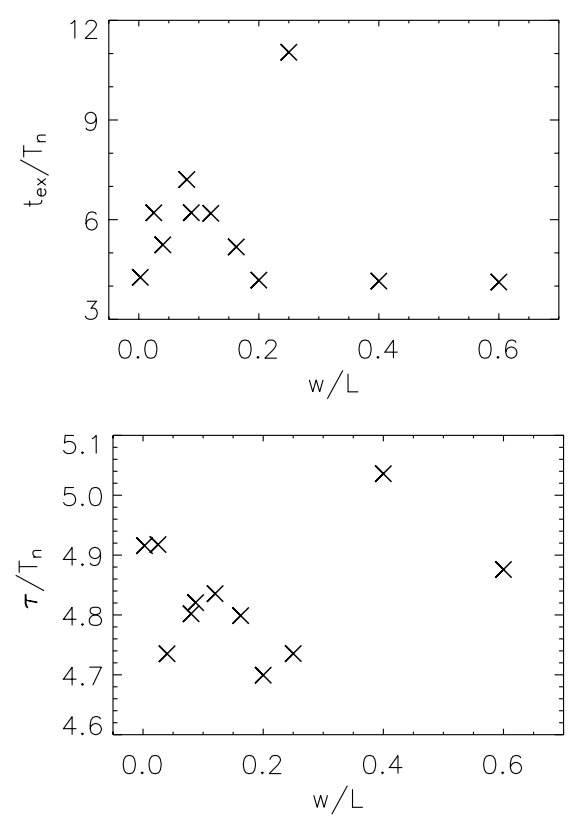

Fig. 9. Normalized fundamental standing wave $(n=1)$ excitation time $t_{\mathrm{ex}} / T_{n}$ (top panel), and damping time $\tau / T_{n}$ (bottom panel) vs. pulse width $w$ for $d=10^{8}$ and $x_{0}=L / 4$.

function of $x$ of the standing wave spatial profile of Eqs. (13)(15); sufficiently wide pulses adjust faster to the standing waves.

In the case of the second standing mode, its excitation and damping times vary with the pulse width (Table 1). For a very wide pulse the second standing wave is excited more slowely than by narrower pulses, but its damping time is slightly shorter. Note that, even for $w=3 \times 10^{9} \mathrm{~cm}$, the initial pulse is close to the sine function of $x$ and the second, not the fundamental, mode is excited for pulse position $x_{0}=L / 2$.
Table 1. Excitation time $t_{\mathrm{ex}}$ and damping time $\tau$ for two different widths of the pulse in the case of the second $(n=2)$ standing wave for $d=10^{8}$ and $x_{0}=L / 2$.

\begin{tabular}{ccc}
\hline \hline$w / L$ & $t_{\mathrm{ex}}[\mathrm{s}]$ & $\tau[\mathrm{s}]$ \\
\hline 0.025 & 463 & 603 \\
0.6 & 1050 & 565 \\
\hline
\end{tabular}
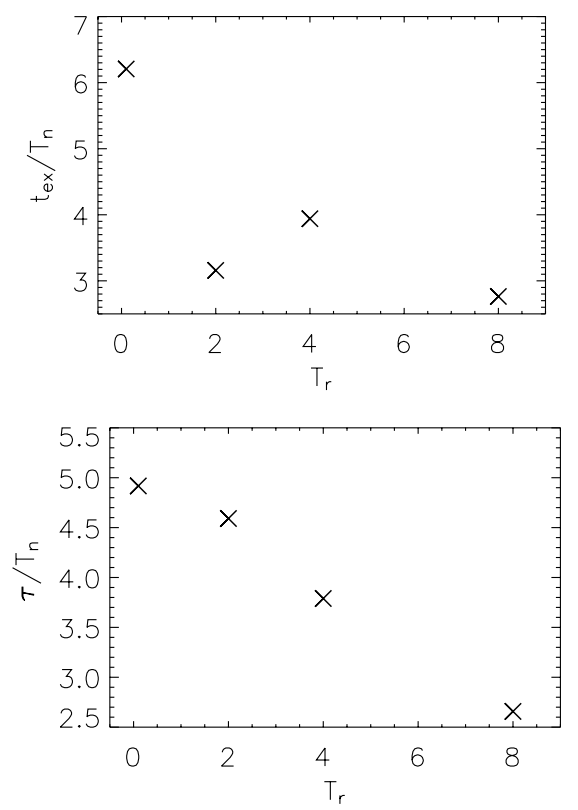

Fig. 10. Normalized fundamental standing wave excitation time (top panel) and damping factor (bottom panel) vs. normalized pulse temperature $T_{\mathrm{r}}$ for $d=10^{8}, w=L / 40$, and $x_{0}=L / 4$.

So far, we have discussed the initial pulses of constant relative temperature $T_{\mathrm{r}}=T_{\mathrm{p}} / T_{\mathrm{c}}=1.1$, where $T_{\mathrm{p}}$ is the temperature of the initial pulse and $T_{\mathrm{c}}$ is the temperature of the corona. This temperature is realized through the choice of pulse amplitudes $A_{\varrho}=0.125 \varrho_{0}\left(x_{0}\right)$ and $A_{\mathrm{p}}=0.25 p_{0}$. It is worth quantifying an influence of $T_{\mathrm{r}}$ on mode excitation time $t_{\mathrm{ex}}$ and damping time $\tau$. To do so, we consider three values of the temperature ratio: $T_{\mathrm{r}}=2\left(A_{\varrho}=0.125 \varrho_{0}\left(x_{0}\right), A_{\mathrm{p}}=1.25 p_{0}\right), T_{\mathrm{r}}=4$ $\left(A_{\varrho}=0.125 \varrho_{0}\left(x_{0}\right), A_{\mathrm{p}}=3.5 p_{0}\right), T_{\mathrm{r}}=8\left(A_{\varrho}=0.125 \varrho_{0}\left(x_{0}\right)\right.$, $\left.A_{\mathrm{p}}=8.0 p_{0}\right)$. Figure 10 summarizes results of the corresponding numerical experiments. According to our expectation a warmer pulse leads to more efficient standing wave excitation as the excitation time declines with $T_{\mathrm{r}}$; a warmer pulse transfers itself in a shorter time to a sinusoidal standing wave profile. As a consequence of higher energy leakage, the standing wave is more rapidly damped for higher values of $T_{\mathrm{r}}$. Given that $T_{\mathrm{r}}$ changes by a factor of 8 , the induced changes in $t_{\mathrm{ex}}$ and $\tau$ are relatively small (factor of 2). As warmer pulses correspond to higher amplitudes of the initial pulses we claim that nonlinearity does not play a vital role in affecting $t_{\mathrm{ex}}$ and $\tau$.

We now compare four cases which correspond to the following loop lengths $L_{0}$ : (a) $L_{0}=L=50 \times 10^{8} \mathrm{~cm}$; (b) $L_{0}=2 L$; (c) $L_{0}=4 L$; and (d) $L_{0}=6 L$. The pulse position was chosen as $x_{0}=L_{0} / 4$. The normalized excitation time decreases with the loop length for narrow pulses (Fig. 11), and the damping time generally grows with loop length $L_{0}$ (right panel). Note 

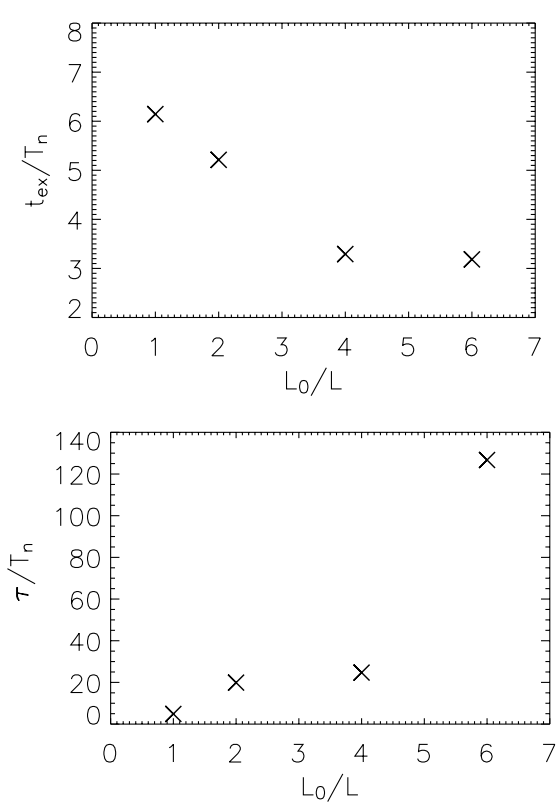

Fig. 11. Normalized fundamental standing wave excitation time (top panel) and damping time (bottom panel) vs. normalized loop length $L_{0}$, for $d=10^{8}, x_{0}=L_{0} / 4$, and $w=L / 40$. Note that $T_{n}$ now corresponds to the expected period for the appropriate loop length.

that since $T_{n} \sim L_{0}$ the results must be considered carefully, e.g. $t_{\mathrm{ex}}$ actually grows with $L_{0}$ (in s).

\subsection{Velocity pulses}

In a similar case to the cases discussed above, the only difference is that to mass density and pressure perturbations we add a non-zero pulse in velocity. We choose and hold fixed $A_{\mathrm{V}}=0.125 c_{\mathrm{s}}\left(x_{0}\right)$. In the case of $x_{0}=L / 4$ the fundamental standing wave is generated at $t_{\mathrm{ex}} \approx 7 T_{1}$, which is a little larger than in the case of $A_{\mathrm{V}}=0$ (Fig. 12). The damping time attains a value of $\tau \approx 5.5 T_{1}$, which is $10 \%$ higher than without the velocity pulse. From this result we conclude that the fundamental mode is weakly damped while triggered by the velocity perturbations.

In the case of both $x_{0}=L / 2$ and the harmonic standing wave (crosses) the excitation time is larger than in the case of $A_{\mathrm{V}}=0$, while the damping time becomes a bit smaller than without the velocity pulse. The harmonic standing wave is excited in a longer lapse of time and is stronger damped while being excited by a velocity pulse.

\subsection{Perturbations of non-ideal plasma}

Next we consider several cases of non-ideal plasma with different effects switched on. In our approach we neglect background heating, which has been used by Nakariakov et al. (2004) to keep background temperature at the coronal value of $\sim 1 \mathrm{MK}$. Instead, we direct most of our attention to the effect of nonideal plasma on wave propagation. Table 2 summarizes all the results that are obtained for the width of the pulse $W=L / 40$ and its initial position $x_{0}=L / 4$.
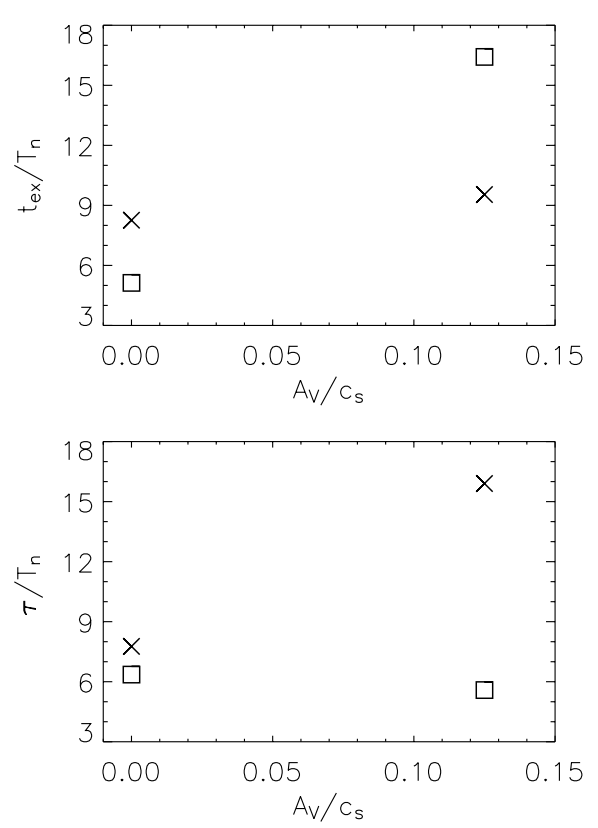

Fig. 12. Normalized fundamental standing wave excitation time (left panel) and damping time (right panel) vs. the velocity amplitude of the initial pulse, $A_{\mathrm{V}}$, for $d=10^{8}, w=L / 40, x_{0}=L / 4$ (squares), and $x_{0}=L / 2$ (crosses).

We discuss in some detail the case of thermal conduction, heating, and cooling acting simultaneously. In this case we do not observe a pure standing wave but instead a packet of waves with largest contribution from the fundamental standing wave (left bottom panel of Fig. 13). Mass density collected at $x_{\mathrm{d}}=$ $L / 4$ exhibits oscillations that decay in time. The mean value of the mass density grows in time as a result of thermal conduction (right top panel of Fig. 13). Velocity spectra reveal the mean period of $T_{1}=410 \mathrm{~s}$ (bottom panels of Fig. 13). Note that this value is higher than in the case of the ideal plasma due to a different value of the effective sound speed $c_{\mathrm{s}}^{\text {eff }}=\sqrt{\gamma p / \varrho}$ that results from the cooling and thermal conduction (Fig. 14).

It is noteworthy that thermal conduction acting alone excites a bunch of waves at the loop foot-points where the thermal conduction is mostly effective (not shown). The most significant value of standing wave period is similar to the case without conductivity. Taking both thermal conduction and heating into account leads to a significant increase in the period of the observed standing wave. In the case of thermal conduction switched on, evaporation of a photospheric plasma takes place. It is interesting, however, that it is not continuous but occurs in the form of parcels which propagate out of the photosphere in certain lapses of time with each separate from the other.

This scenario can be explained as follows. Photospheric plasma gets heated due to thermal conduction. As this heated material is denser than a coronal plasma, a sufficient amount of heating is needed for a parcel of the photospheric gas to attain enough pressure to leave the photosphere.

\section{Summary}

In this paper we have considered the excitation and damping of slow standing waves in a solar coronal loop that is 
Table 2. Standing wave period $T$, excitation time $t_{\mathrm{ex}}$, and damping time $\tau$ for various run parameters in cgs units. The sign - means that a value could not be evaluated.

\begin{tabular}{ccccccccccc}
\hline \hline$A_{\varrho}$ & $A_{\mathrm{p}}$ & $A_{\mathrm{V}}$ & $d$ & $c_{\mathrm{s}}\left[\mathrm{cm} \mathrm{s}^{-1}\right]$ & $\mathcal{H}$ & $\mathcal{L}$ & $\sigma$ & $T[\mathrm{~s}]$ & $t_{\mathrm{ex}}[\mathrm{s}]$ & $\tau[\mathrm{s}]$ \\
\hline$\neq 0$ & $\neq 0$ & $=0$ & $10^{8}$ & $0.35 \times 10^{8}$ & $=0$ & $=0$ & $=0$ & 254 & 1578 & 1250 \\
$\neq 0$ & $\neq 0$ & $=0$ & $10^{8}$ & $0.35 \times 10^{8}$ & $\neq 0$ & $=0$ & $=0$ & 208 & 915 & 986 \\
$\neq 0$ & $\neq 0$ & $=0$ & $10^{8}$ & $0.35 \times 10^{8}$ & $\neq 0$ & $\neq 0$ & $=0$ & 208 & 916 & 998 \\
$=0$ & $=0$ & $=0$ & $10^{8}$ & $0.35 \times 10^{8}$ & $\neq 0$ & $\neq 0$ & $=0$ & 208 & 919 & 1009 \\
$=0$ & $=0$ & $=0$ & $10^{8}$ & $0.35 \times 10^{8}$ & $=0$ & $=0$ & $\neq 0$ & - & - & - \\
$\neq 0$ & $\neq 0$ & $=0$ & $10^{8}$ & $0.35 \times 10^{8}$ & $=0$ & $=0$ & $\neq 0$ & 500 & 1291 & 2289 \\
$\neq 0$ & $\neq 0$ & $=0$ & $10^{8}$ & $0.35 \times 10^{8}$ & $\neq 0$ & $=0$ & $\neq 0$ & 556 & 1133 & 1178 \\
$=0$ & $=0$ & $=0$ & $10^{4}$ & $0.35 \times 10^{8}$ & $=0$ & $=0$ & $\neq 0$ & 278 & - & - \\
$\neq 0$ & $\neq 0$ & $=0$ & $10^{4}$ & $0.35 \times 10^{8}$ & $=0$ & $=0$ & $\neq 0$ & 455 & - & - \\
$\neq 0$ & $\neq 0$ & $=0$ & $10^{8}$ & $0.1 \times 10^{8}$ & $=0$ & $=0$ & $=0$ & 882 & 4627 & 3826 \\
$\neq 0$ & $\neq 0$ & $=0$ & $10^{8}$ & $0.1 \times 10^{8}$ & $=0$ & $=0$ & $\neq 0$ & 909 & 1113 & 3930 \\
$\neq 0$ & $\neq 0$ & $=0$ & $10^{8}$ & $0.35 \times 10^{8}$ & $\neq 0$ & $\neq 0$ & $\neq 0$ & 400 & 827 & 2170 \\
\hline
\end{tabular}
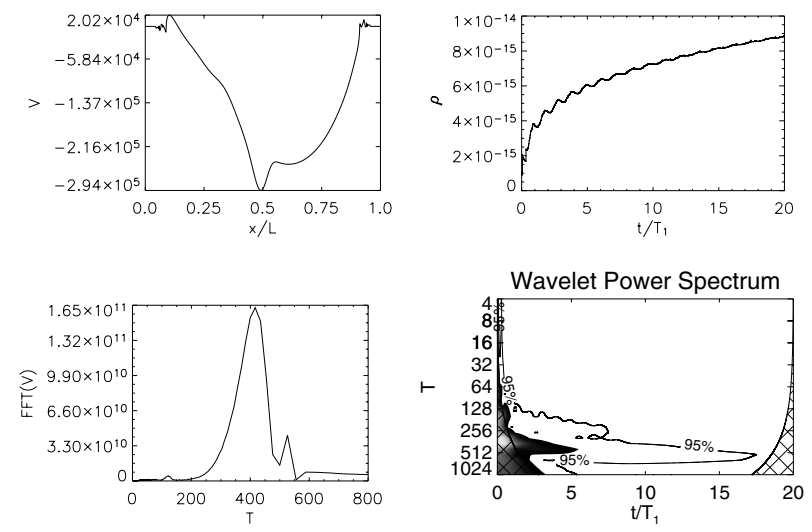

Fig. 13. A spatial velocity profile at $t \approx 12 T_{1}$ (left top panel), the corresponding time-characteristics of mass density signal (right top panel), the Fourier spectrum (left bottom panel), and the wavelet spectrum (right bottom panel) for $d=10^{8}, w=L / 40, x_{0}=L / 4, \sigma \neq 0$, $\mathcal{H} \neq 0, \mathcal{L} \neq 0, A_{\varrho} \neq 0, A_{\mathrm{p}} \neq 0$, and $A_{\mathrm{V}}=0$.

approximated by a one-dimensional plasma. Our model implements optionally thermal conduction, heating, cooling, and impulses in plasma quantities. The thermal conductivity follows the formulation of Spitzer (1962). The phenomenological heating source term is localized in space and time. Cooling is due to radiative losses from an optically thin plasma. A Fast Fourier transform and wavelet analysis of temporal wave profiles have shown that in a loop the fundamental or harmonic standing slow waves are excited depending on the location of the trigger. We worked out a simple criterion for the presence of a standing wave based on a phase shift between perturbed mass density $\delta \varrho$ and velocity $V$. The time required for a standing wave to settle in a loop from a hot initial pulse is of the order of a dozen or so wave periods and varies, depending on such parameters of the plasma as the initial location of the pulse, the density contrast, or the pulse width. In the case of a wide and strong pulse, as well as of heated plasma, this time-scale is shorter.

The one-dimensional model we have developed obviously suffers from several drawbacks. Its main shortcoming is that
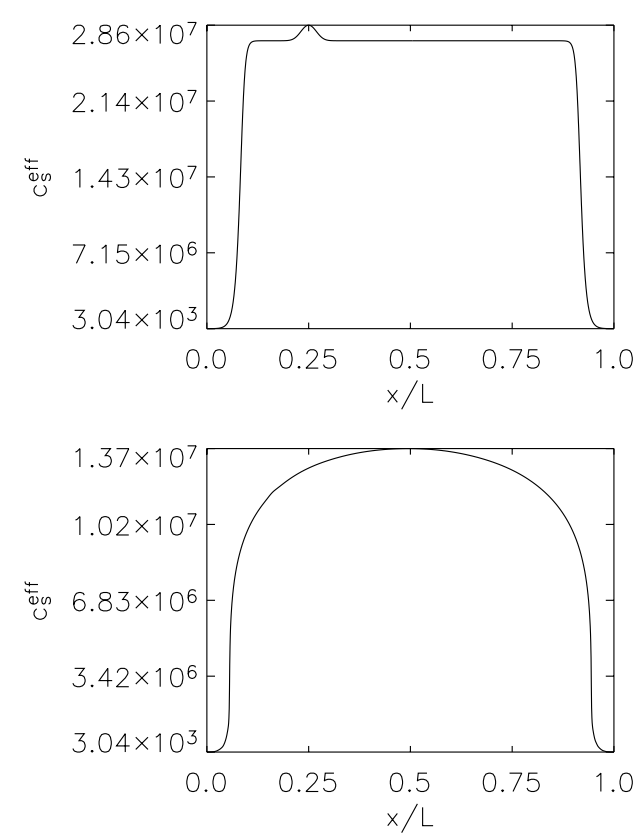

Fig. 14. Effective sound speed at $t=0$ (top panel) and $t=5000 \mathrm{~s}$ (bottom panel) in the case of Fig. 13.

it only takes slow magnetosonic waves into account and neglects the presence of fast and Alfvén waves in the system. As a consequence, this model excludes several likely damping mechanisms that were already suggested in the literature. However, these neglected effects are believed to be unimportant as far as the scenario of slow wave excitation and damping are concerned. More realistic models of slow wave development are currently under development and the results will be reported elsewhere.

Acknowledgements. The authors express their thanks to the anonymous referee and to Dr. Luigi Nocera for their constructive comments on an earlier draft of this paper. The FLASH code is provided by ASCI (Alliances Center for Astrophysical Thermonuclear Flashes) at the University of Chicago. Wavelet software is disseminated by C. Torrence and G. Compo, and is available at 
URL http://paos.colorado.edu/research/wavelets. M.S.'s and K.M.'s work was financially supported by a grant from the State Committee for Scientific Research Republic of Poland, with KBN grant No. 2 PO3D 01625.

\section{References}

Aschwanden, M., Fletcher, L., Schrijver, C., \& Alexander, D. 1999, ApJ, 520, 880

Curdt, W., Wang, T. J., Dammasch, T. E., \& Solanki, S. K. 2003, Hvar Obs. Bull., 27, 83

De Moortel, I., \& Hood, A. W. 2003, A\&A, 408, 755

De Moortel, I., Hood, A. W., \& Ireland, J. 2002a, A\&A, 381, 311

De Moortel, T., Hood, A. W., Ireland, J., \& Walsh, R. W. 2002b, Sol. Phys., 209, 89

Fryxell, B., Olson, K., Ricker, P., et al. 2000, ApJS, 131, 273

Nakariakov, V. M., Ofman, L., DeLuca, E. E., Roberts, B., \& Davila, J. M. 1999, Science, 285, 862

Nakariakov, V. M., Verwichte, E., Berghmans, D., \& Robbrecht, E. 2000, A\&A, 362, 1151
Nakariakov, V. M., Tsiklauri, D., Kelly, A., Arber, T. D., \& Aschwanden, M. J. 2004, A\&A, 414, L25

Ofman, L. 2002, ApJ, 568, L135

Ofman, L., \& Wang, T. J. 2002, ApJ, 580, L85

Ofman, L., \& Aschwanden, M. 2002, ApJ, 576, L153

Ofman, L., Nakariakov, V. M., \& Sehgal, N. 2000, ApJ, 533, 1071

Roberts, B. 2000, Sol. Phys., 193, 139

Ruderman, M. S., \& Roberts, B. 2002, ApJ, 577, 475

Spitzer, L. 1962, Physics of fully ionized gases (New York: Wiley)

Torrence, C., \& Compo, G. P. 1998, Bull. Amer. Meteor. Soc., 79, 61

Tsiklauri, D., Nakariakov, V. M., Arber, T. D., \& Aschwanden, M. J. 2004, A\&A, 422, 351

Van Doorsselaere, T., Debosscher, A., Andries, J., \& Poedts, S. 2004, A\&A, 424, 1065

Wang, T. J., Solanki, S. K., Curdt, W., Innes, D. E., \& Dammasch, I. E. 2002, ApJ, 574, L101

Wang, T. J., Solanki, S. K., Innes, D. E., Curdt, W., \& Marsch, E. 2003a, A\&A, 402, L17

Wang, T. J., Solanki, S. K., Curdt, W., et al. 2003b, A\&A, 406, 1105

Zingale, M., Dursi, L. J., ZuHone, J., et al. 2002, ApJS, 143, 539 